\title{
Characterization of deep level traps responsible for isolation of proton implanted GaAs
}

\author{
H. Boudinova) and A. V. P. Coelho \\ Instituto de Física, Universidade Federal do Rio Grande do Sul, 91501-970 Porto Alegre, RS, Brazil \\ H. H. Tan and C. Jagadish \\ Department of Electronic Material Engineering, Research School of Physical Sciences and Engineering, \\ The Australian National University, Canberra, ACT 0200, Australia
}

(Received 12 September 2002; accepted 19 December 2002)

\begin{abstract}
Deep level transient spectroscopy was employed to determine the electrical properties of defects induced in metalorganic chemical-vapor deposition grown $n$-type and $p$-type GaAs during proton bombardment. Thermal stability of these defects was investigated and correlation with defects responsible for isolation of GaAs by ion bombardment was discussed. The annealing temperature region $\left(220-250{ }^{\circ} \mathrm{C}\right)$ is similar to proton isolated GaAs below the threshold dose for complete isolation. At least four of the five traps observed in $n$-type GaAs are not simple interstitial-vacancy pairs. For $p$-type GaAs we have observed an unknown level with apparent energy of $\sim 0.64 \mathrm{eV}$. (C) 2003 American Institute of Physics. [DOI: 10.1063/1.1554761]
\end{abstract}

\section{INTRODUCTION}

Ion implantation is an essential process for production of modern III-V compound semiconductor devices and circuits and has been proven as a successful method to convert a conductive layer into a highly resistive one. ${ }^{1,2}$ Due to its simplicity, precise depth control and compatibility with planar technologies, ion implantation is a potential alternative for mesa etching. Selective masking of the semiconductor surface with photoresist followed by ion irradiation is an efficient and practical way to isolate closely spaced devices. ${ }^{3}$ Irradiation of GaAs by ions results in resistivity increase as a consequence of free carrier removal by trapping at defects introduced by the irradiation. ${ }^{4}$ Ion beam isolation is often employed in the processing of GaAs devices, using light ions such as proton, helium, etc. Proton irradiation has been used successfully to isolate GaAs field effect transistors, high electron mobility transistors and heterojunction bipolar transistors. ${ }^{4}$ Waveguiding regions in optoelectronic devices were fabricated by controllable introduction of defects using proton bombardment. ${ }^{5}$ Thus, the understanding of defect creation, carrier trapping and thermal stability of the traps is essential in achieving better device performance.

The threshold dose to convert a conductive GaAs layer to a highly resistive one was found to closely correlate with the estimated number of lattice atom replacements along the depth of the doped layer. ${ }^{6}$ The threshold doses are quite similar for irradiation conducted at room temperature or at $220{ }^{\circ} \mathrm{C}^{7}$ in spite of the enhanced dynamic annealing in the later case. Antisite defects originating at the replacement collisions and their related defect complexes are considered to be the carrier trapping centers, ${ }^{6}$ by virtue of their low sensitivity to dynamic annealing. The stability of the isolation during postirradiation thermal annealing increases progres-

\footnotetext{
a) Author to whom correspondence should be addressed; electronic mail: henry@if.ufrgs.br
}

sively with the increase of the implanted dose and for a constant dose a higher stability of the isolation occurs in samples having the lower original sheet carrier concentration. ${ }^{8}$

Isolation of $n$-type and $p$-type GaAs layers of similar original sheet carrier concentration is attained after implantation of identical ion doses. ${ }^{9}$ This result was explained assuming that conduction electrons and holes are trapped by acceptor and donor centers, these centers being related to $\mathrm{Ga}$ and As antisite complex defects, respectively. Because the displacement energies of $\mathrm{Ga}$ and As are almost equivalent, ${ }^{10}$ similar concentrations of both antisite defects are formed in the collision cascades and hence similar concentrations of electron and hole traps are created.

Electron irradiation damage in GaAs has been studied extensively using a variety of techniques. Electrical resistivity, Hall, and $C-V$ measurements have been used to study the production of damage at different energies and the annealing kinetics of the defects. ${ }^{11,12}$ Deep level transient spectroscopy (DLTS) measurements have been widely employed, ${ }^{13,14}$ and their use has led to a fairly detailed model of many of the traps introduced into $n$ - and $p$-type GaAs by electron irradiation. ${ }^{15,16}$ The traps identified in proton irradiated $\mathrm{GaAs}^{17}$ include most of those, found in electron irradiated materials, either after irradiation or after irradiation and annealing. Some of these traps, which have not been observed in electron irradiated GaAs, are associated with defects which are more complex than simple interstitial-vacancy pairs.

In the present work, the electrical properties of defects induced in metalorganic chemical-vapor deposition grown $n$-type and $p$-type GaAs during proton bombardment were investigated. DLTS measurements were used in an attempt to correlate the deep level center annealing process with the thermal stability of $n$-type and $p$-type GaAs, isolated by proton irradiation. The deep levels measured in this work were 
compared with electron bombardment induced deep levels, published previously.

\section{EXPERIMENTAL TECHNIQUES}

Epitaxial layers of $n$-type and $p$-type GaAs were grown on epi-ready vertical gradient freeze Si-doped (carrier concentration of $2 \times 10^{18} \mathrm{~cm}^{-3}$ ) and $\mathrm{Zn}$-doped (carrier concentration of $\left.6-8 \times 10^{18} \mathrm{~cm}^{-3}\right)\langle 100\rangle \mathrm{GaAs}$ with $2^{\circ}$ off normal toward the (110) using the Australian National University (ANU) metalorganic chemical-vapor deposition (MOCVD) reactor. Trimethylgallium was used as the group III source with $\mathrm{H}_{2}$ as the carrier gas and arsine was used as the group V source. Epitaxial layers of $6-\mu \mathrm{m}$-thick GaAs were grown to produce $n / n^{+}$and $p / p^{+}$samples. Electron concentration of $1.0 \times 10^{16} \mathrm{~cm}^{-3}$ in the $n$-type samples and hole concentration of $1.1 \times 10^{16} \mathrm{~cm}^{-3}$ in $p$-type samples were obtained by using diluted silane and dimethylzinc, respectively. Irradiation was carried out using the ANU 1.7 MV tandem accelerator with $600 \mathrm{keV} \mathrm{H}^{+}$ions from $\mathrm{TiH}$ source. During irradiation, the samples were held nominally at room temperature and tilted $7^{\circ}$ from the beam axis to minimize channeling effects. The ion fluence was $1 \times 10^{11} \mathrm{~cm}^{-2}$ with a constant flux of $\sim 0.5$ $\mathrm{nA} / \mathrm{cm}^{2}$. After irradiation, the samples were submitted to rapid thermal annealing (RTA) for $1 \mathrm{~min}$ in the temperature range $100-600{ }^{\circ} \mathrm{C}$ in $\mathrm{Ar}$ atmosphere. The annealing steps were performed with the irradiated face in close proximity with fresh GaAs dummy wafer to prevent excessive loss of As from the surface. All samples were then chemically cleaned, including a final dip in diluted hydrochloric acid to remove any surface oxide. Immediately after cleaning, the samples were loaded into a vacuum chamber to form Schottky barrier contacts by thermal evaporation of $\mathrm{Au}$ at a base pressure of $\sim 2 \times 10^{-6}$ Torr. The deposited film thickness was $\sim 100 \mathrm{~nm} . C-V$ and DLTS measurements were carried out in experimental setup which consists of a $1 \mathrm{MHz}$ high-speed capacitance bridge, a pulse generator, and a cryostat $(77-373 \mathrm{~K})$. The setup is controlled via computer interface, except for the temperature regulator, which is operated manually. The measured transients are transferred to and stored in the memory of the computer together with the sample temperature. The apparent energy level $\left(E_{a}\right)$ in the band gap and apparent capture cross section $\left(\sigma_{a}\right)$ of defects were determined from Arrhenius plots of $\tau T^{2}$ vs $1 / T$, where $1 / \tau$ is the emission rate at a temperature $T$.

\section{RESULTS AND DISCUSSION}

Figure 1 shows the DLTS spectra for as-grown, irradiated and various temperature annealed $n$-type [Fig. 1(a) and $p$-type [Fig. 1(b)] samples for a rate window of $0.98 \mathrm{~s}^{-1}$. Five main peaks were observed for $n$-type (called $P n 1, P n 2$, $P n 3, P n 4$, and $P n 5)$ and four for $p$-type samples $(P p 1, P p 2$, $P p 3$, and $P p 4)$ in the scanned temperature interval (77-373 K). We have shown in Fig. 2 the fitting of DLTS spectra with a sum of a number of Lorenzian peaks. Using this technique we have separated the peaks better, especially for the case of a small peak overlapping with a large one [see Fig. 2(a)]. This method permits one to obtain the maximum temperatures $\left(T_{\max }\right)$ of the peaks more precisely.
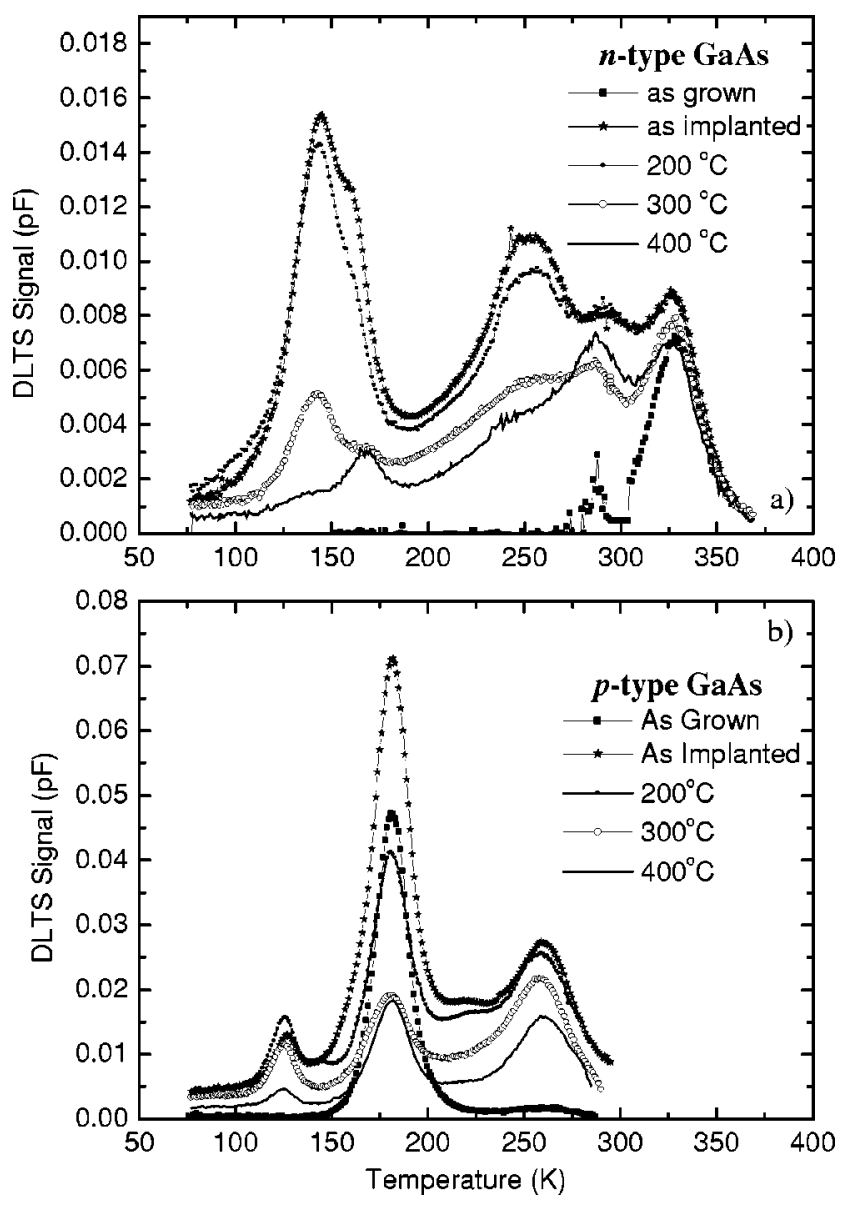

FIG. 1. DLTS spectra (rate window $0.98 \mathrm{~s}^{-1}$ ) showing the evolution of the deep levels with the annealing temperature after $\mathrm{H}^{+}$implantation to a dose of $1 \times 10^{11} \mathrm{~cm}^{-2}$ for $n$-type (a) and $p$-type (b) GaAs.

The trap signatures of the observed deep levels (experimental points) are compared with those of known deep level centers (solid lines) as shown by the plot in Fig. 3. The signature of $P n 1$ is similar to that of $E L 2$ level. There are presently two microscopic models of EL2. One is that $E L 2$ is an intrinsic and complex lattice defect with an arsenic antisite $\mathrm{As}_{\mathrm{Ga}}$ as a core and arsenic interstitial $\mathrm{As}_{i}$ as a neighbor, forming pair as a result of a Coulomb interaction. ${ }^{18}$ The other is that EL2 is simply an isolated arsenic antisite $\mathrm{As}_{\mathrm{Ga}}$ or gallium vacancy-arsenic interstitial $\left(\mathrm{V}_{\mathrm{Ga}} \mathrm{As}_{i}\right)$ complex. ${ }^{19}$

$P n 2$ and $P n 3$ peaks were resolved, using the method shown in Fig. 2. From the trap signature of $P n 2$, it is likely that it corresponds to two different peaks with similar apparent energy. We can note this peak in as-grown material [see Fig. 1(a)]. It is known like $E$ level $^{18}$ and was measured in as-grown MOCVD material. After irradiation and during post-irradiation annealing $P n 2$ is transformed into the $P 3$ level well known from the literature. ${ }^{14}$

Many works claim that the well known traps $E 1-E 5^{14}$ in $n$-type GaAs have to be ascribed to simple defects, i.e., that they are produced by the displacements of one single atom with a threshold energy of $\sim 10 \mathrm{eV}$. We would like to mention here that the threshold energy for the replacement collision to occur is the same. Consequently, during a knock on with transferred energy of $10-15 \mathrm{eV}$, it is possible to create 

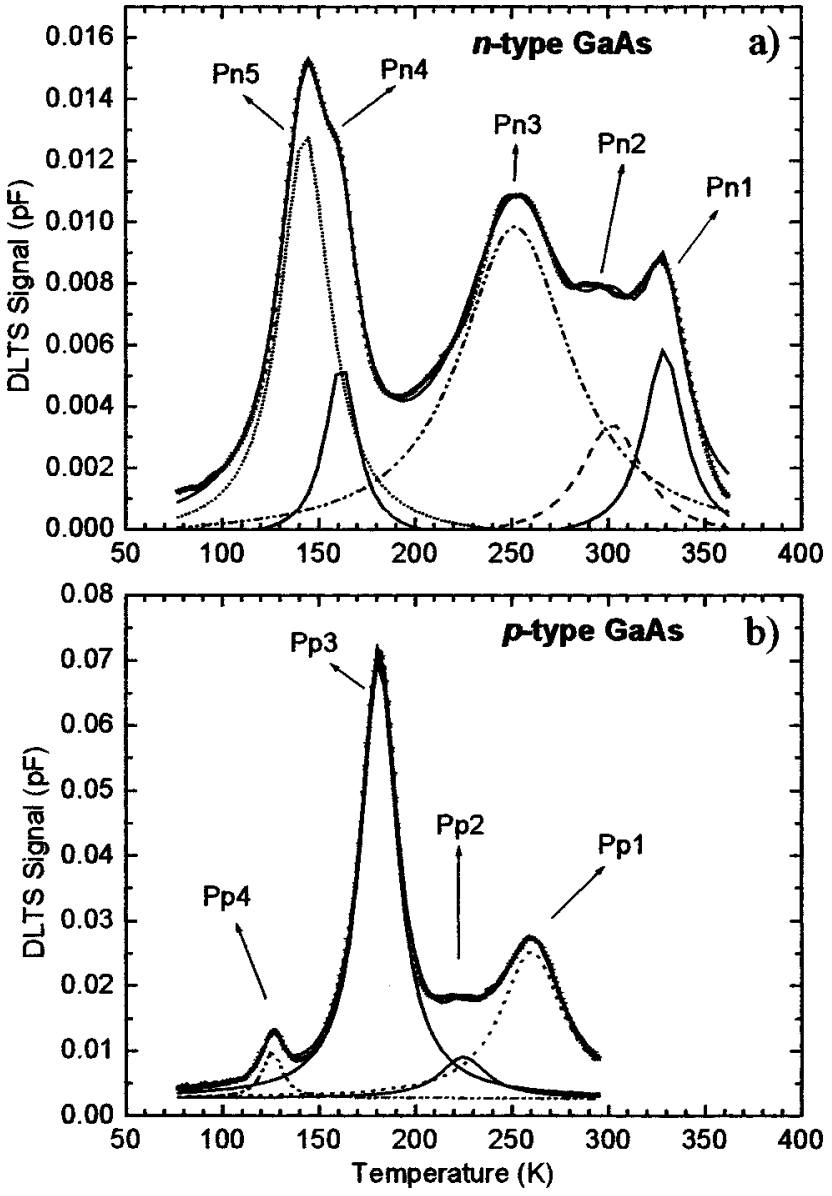

FIG. 2. Fitting of DLTS spectra with a sum of Lorenzian peaks for $n$-type (a) and $p$-type (b) GaAs.

not only simple defects (like $\mathrm{V}_{\mathrm{As}}, \mathrm{V}_{\mathrm{Ga}}, \mathrm{Ga}_{i}, \mathrm{As}_{i}$ ), but also antisite related defect complexes (for example, $\mathrm{As}_{\mathrm{Ga}}+\mathrm{V}_{\mathrm{As}}+\mathrm{Ga}_{i}$ or $\left.\mathrm{Ga}_{\mathrm{As}}+\mathrm{V}_{\mathrm{Ga}}+\mathrm{As}_{i}\right)$. Taking into account the different mobility for different parts of these defects at the temperature of bombardment and even the ion irradiation current density influence, we can explain the creation of various complex defects. For example the peak Pn3 in Fig. 3 has a signature of E4 defect, ${ }^{20}$ which is believed to be an $\mathrm{As}_{\mathrm{Ga}}+\mathrm{V}_{\mathrm{As}}$ defect complex. ${ }^{15}$ The small Pn4 peak at $\sim 170 \mathrm{~K}$ has a trap signature similar to that of $P 1,{ }^{14}$ which must be a complex defect, because its concentration increases with the annealing temperature [see Figs. 1(a) and 4(a)]. The last peak $P n 5$ has a signature close to that of E3, which is believed to be a simple defect, ${ }^{16}$ but it was shown ${ }^{17}$ that this peak is due to two different traps, one of them being E3 and the other a complex defect. All the observed deep levels in $n$-type GaAs are listed in Table I.

The main difference between electron and proton irradiated $n$-type GaAs is that in the later case more complex defects are observed (eventually antisite related), with respect to the simple defects in the former case. There is one more issue, related to traps and isolation of GaAs, which we would like to discuss. To the best of our knowledge, there is no published DLTS result discussing the Ga antisite as a possible trap, even though electrically $\mathrm{Ga}_{\mathrm{As}}$ is a good candidate for trapping electrons. The common explanation in the
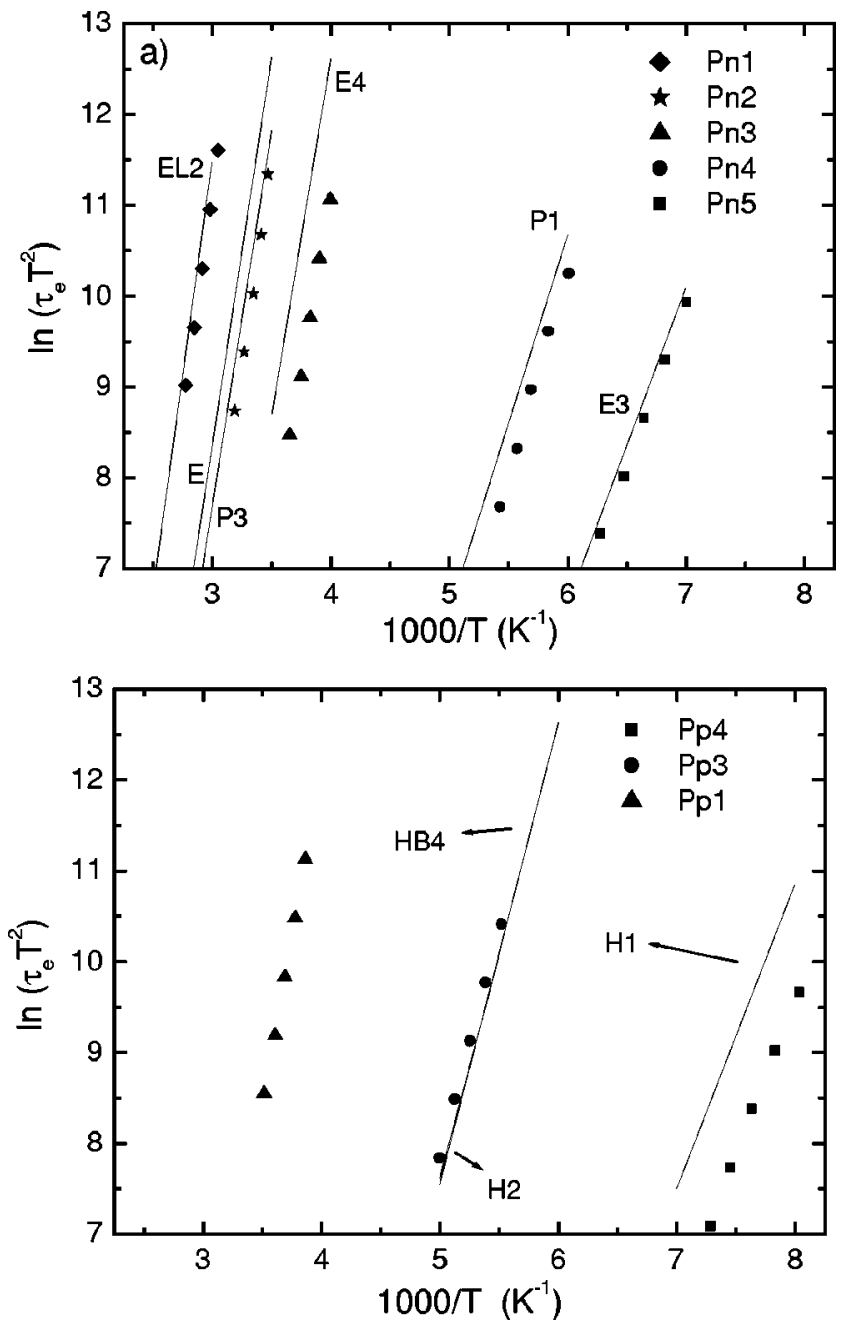

FIG. 3. Comparison of the trap signatures for the observed deep levels (experimental points) and those from the literature (solid lines) for $n$-type (a) and $p$-type (b) GaAs (see Tables I and II).

literature is that due to the high mobility of the point defects in the Ga sublattice during irradiation, these defects are not stable. This is true for $\mathrm{V}_{\mathrm{Ga}}$ and $\mathrm{Ga}_{i}$, but the $\mathrm{Ga}_{\mathrm{As}}$ like a point defect without the presence of other point defects in close proximity could be stable and its annealing depends on the flux of other point defects. In this way the Ga antisite defect could participate in the isolation process, too.

In $p$-type GaAs the situation is different. It is well accepted that creation of defect complexes is possible even during low dose electron irradiation. ${ }^{15}$ DLTS spectra for proton irradiated $p$-type GaAs samples [Figs. 1(b) and Fig. 3(b)] have not previously been reported. The signature of $P p 1$ is not known from the literature and presents characteristic energy level of $E_{T}-E_{V} \sim 0.64 \mathrm{eV}$. A very broad $P p 2$ peak is seen around $210-230 \mathrm{~K}$. It is interesting to note that this is the temperature range where the so-called $\mathrm{U}$ band is commonly observed in irradiated $n$-type samples. ${ }^{21}$ From the trap signature of $P p 3$, it is possible to correlate this peak with two different trap levels, having similar apparent parameters. $P p 3$ exists in the as-grown material [Fig. 1(b)] and corresponds to $H B 4$ observed in epitaxial layers ${ }^{18}$ or the so-called $H 2$ level, observed in electron irradiated $p$-type GaAs. ${ }^{22}$ Pp4 apparent 

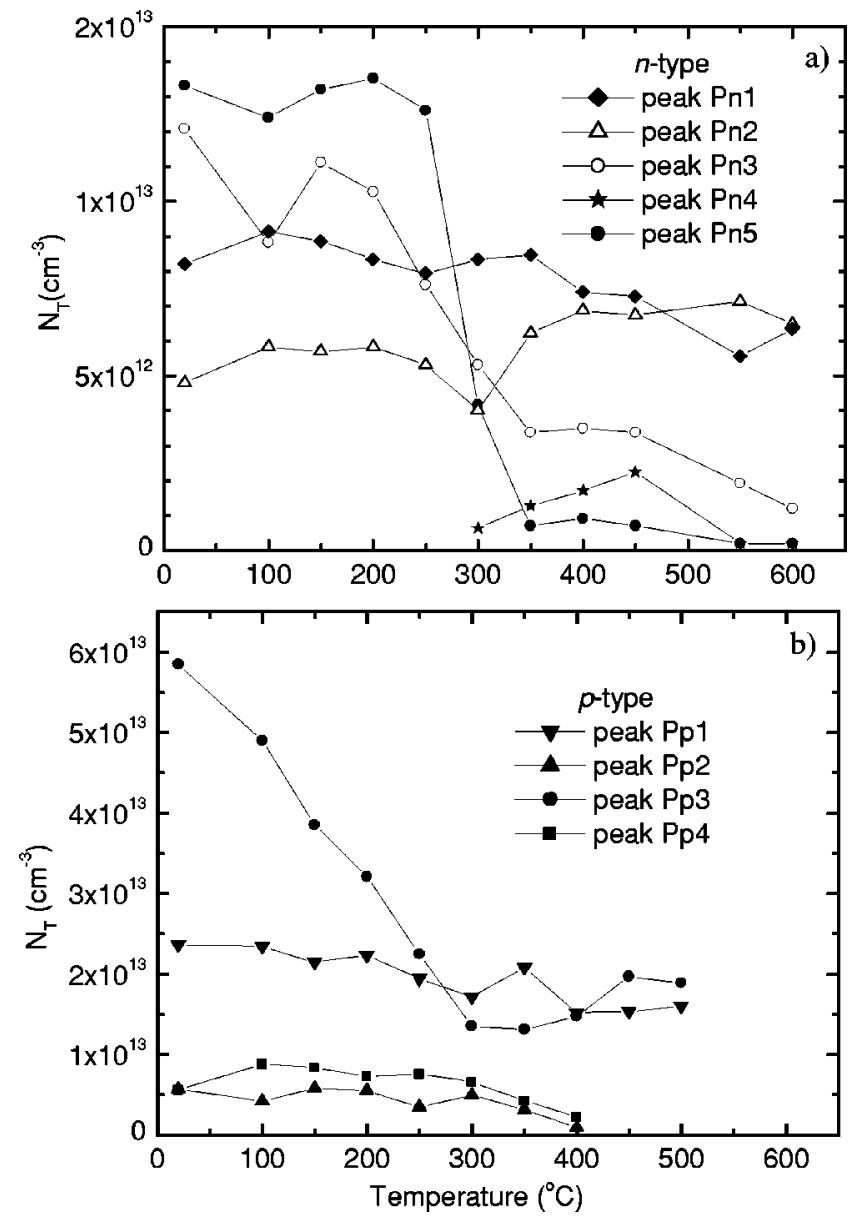

FIG. 4. Thermal stability of the measured deep levels in $n$-type (a) and p-type (b) GaAs after 1 min RTA annealing in Ar atmosphere.

energy is similar to $H 1^{16}$ trap level, known as a Frenkel pair defect. Table II summarizes the observed deep levels in p-type GaAs.

We consider now the thermal annealing of the measured defects. Figure 4 shows the dependence of the trap concentrations versus the annealing temperature. The levels $P n 3$ and $P n 5$ have similar annealing behavior. It is known ${ }^{16}$ that $E 3$ and $E 4$ levels anneal at the same characteristic temperature (so-called stage III). The similarity in the annealing behavior between $P n 5$ and $P n 3$ suggests that the mentioned complex defect constituting $P n 5$ has the same structure like $E 4$. Maybe these two levels correspond to the same defect, but with different charge state (two different charge states of $\mathrm{As}_{\mathrm{Ga}}+\mathrm{V}_{A s}$ are considered in Ref. 23). Another interesting feature observed in Fig. 4(a) is that the concentration of $P n 2$ and $P n 4$ (levels $P 3$ and $P 1$, respectively) is increasing for

TABLE I. Deep traps in $n$-type GaAs.

\begin{tabular}{lcccc}
\hline \hline Peak & $E_{\text {na }}(\mathrm{eV})$ & $\sigma_{\text {na }}\left(\mathrm{cm}^{2}\right)$ & Defect & Reference \\
\hline Pn1 & 0.82 & $2 \times 10^{-13}$ & EL2 & 18 \\
Pn2 & 0.80 & $6 \times 10^{-12}$ & P3 & 14 \\
Pn3 & 0.67 & $2 \times 10^{-12}$ & E4 & 19 \\
Pn4 & 0.39 & $7 \times 10^{-14}$ & P1 & 14 \\
Pn5 & 0.30 & $8 \times 10^{-15}$ & E3 & 16 \\
\hline \hline
\end{tabular}

TABLE II. Deep traps in $p$-type GaAs.

\begin{tabular}{lcccc}
\hline \hline Peak & $E_{\text {na }}(\mathrm{eV})$ & $\sigma_{\text {na }}\left(\mathrm{cm}^{2}\right)$ & Defect & Reference \\
\hline$P p 1$ & 0.64 & $3 \times 10^{-14}$ & $?$ & $\ldots$ \\
$P p 3$ & 0.42 & $1 \times 10^{-14}$ & $H B 4, H 2$ & 18,21 \\
$P p 4$ & 0.29 & $3 \times 10^{-14}$ & $H 1$ & 16 \\
\hline \hline
\end{tabular}

temperatures higher than $250{ }^{\circ} \mathrm{C}$ in agreement with the data of Pons, Mircea, and Bourgoin. ${ }^{14}$ data. The Pn1 (EL2) level does not change considerably during the annealing. Figure 4(b) shows the annealing behavior of traps observed in $p$-type GaAs. All the peaks decrease slowly with the temperature, but the most significant decreasing is of the peak $P p 3$ (H2), where for temperatures above $200^{\circ} \mathrm{C}$, the concentration of this level becomes lower than in the as-grown sample.

Figure 5 compares the temperature dependence for the sum of all $n$-type and $p$-type traps. It is worth mentioning here that we did not observe $E 1, E 2$ and $H 0$ levels, ${ }^{18}$ due to the restricted temperature interval $(77-373 \mathrm{~K})$ of our DLTS measurements. We observed that the main annealing temperature region is $220-250{ }^{\circ} \mathrm{C}$ (named stage $\mathrm{III}^{16}$ ). This region coincides with the characteristic temperature of thermal stability for proton-isolated $n$-type and $p$-type $\mathrm{GaAs}^{8,9}$ with doses below the threshold dose for complete isolation. Using this correlation, we can conclude that the measured deep level defects in the present work are part of the traps responsible for the isolation in ion bombarded GaAs.

\section{CONCLUSIONS}

The results shown in this work lead to the conclusion that not all the traps, observed in electron irradiated $n$-type and $p$-type GaAs can be identified in proton irradiated epitaxial layers. Some traps are detected in proton irradiated GaAs, which are not present in electron irradiated GaAs. For $p$-type GaAs we have observed an unknown level with apparent energy of $\sim 0.64 \mathrm{eV}$. At least four of the five traps observed in $n$-type GaAs are not simple defects. Strong evi-

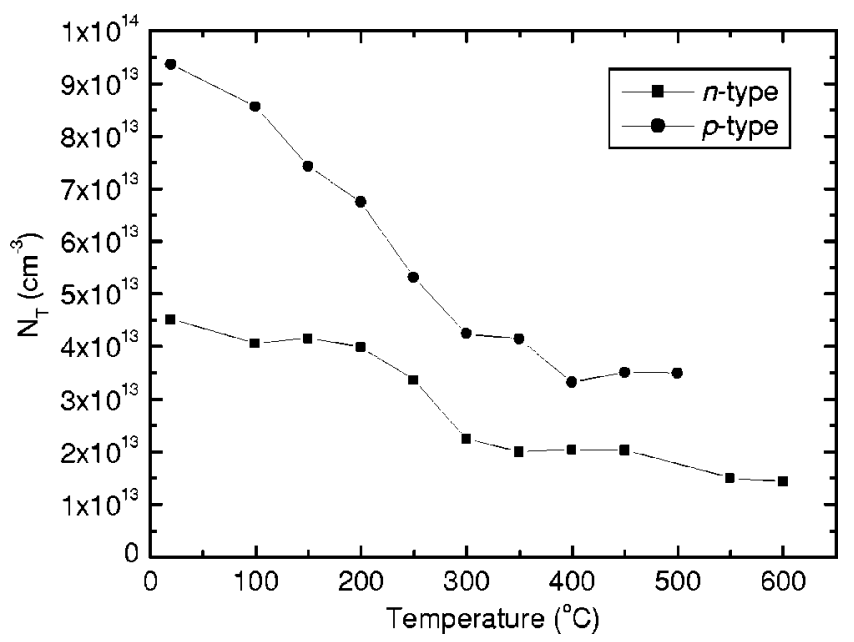

FIG. 5. Temperature evolution for the sum of all measured deep levels in $n$-type and $p$-type GaAs. 
dence is found that most of the defects responsible for trapping during ion isolation are related to antisite complex defects. The characteristic annealing temperature region (220$250{ }^{\circ} \mathrm{C}$ ) for these defects is similar to proton isolated $\mathrm{GaAs}$ below the threshold dose for complete isolation.

\section{ACKNOWLEDGMENTS}

This work was partially supported by Conselho Nacional de Desenvolvimento Científico e Tecnológico (CNPq), and Fundação de Amparo à Pesquisa do Estado do Rio Grande do Sul (FAPERGS). H.H.T. gratefully acknowledges the Australian Research Council for the financial support.

${ }^{1}$ S. J. Pearton, Mater. Sci. Rep. 4, 313 (1990).

${ }^{2}$ S. J. Pearton, Int. J. Mod. Phys. B 7, 4687 (1993).

${ }^{3}$ F. Ren, S. J. Pearton, W. S. Hobson, T. R. Fullowan, J. Lothian, and A. W. Yanof, Appl. Phys. Lett. 56, 860 (1990).

${ }^{4}$ D. V. Morgan and F. H. Eisen, in Gallium Arsenide Materials, Devices, and Circuits, edited by M. J. Howes and D. V. Morgan (Wiley, New York, 1985), p. 161.

${ }^{5}$ T. C. Huang, Y. Chung, L. A. Coldren, and N. Dagli, IEEE J. Quantum Electron. 29, 1131 (1993).

${ }^{6}$ J. P. de Souza, I. Danilov, and H. Boudinov, Appl. Phys. Lett. 68, 535 (1996).
${ }^{7}$ J. P. de Souza, I. Danilov, and H. Boudinov, J. Appl. Phys. 84, 4757 (1998).

${ }^{8}$ J. P. de Souza, I. Danilov, and H. Boudinov, J. Appl. Phys. 81, 650 (1997).

${ }^{9}$ J. P. de Souza, I. Danilov, and H. Boudinov, Radiat. Eff. Defects Solids 147, 109 (1998)

${ }^{10}$ J. W. Corbett and J. C. Bourgoin, in Point Defects in Solids, edited by J. H. Crawford and L. M. Slifkin (Plenum, New York, 1975), p. 118.

${ }^{11}$ L. W. Aukerman and R. D. Graft, Phys. Rev. 127, 1576 (1962).

${ }^{12}$ J. W. Farmer and D. C. Look, Phys. Rev. B 21, 3389 (1980).

${ }^{13}$ D. V. Lang and L. C. Kimmerling, Inst. Phys. Conf. Ser. 23, 581 (1975).

${ }^{14}$ D. Pons, A. Mircea, and J. Bourgoin, J. Appl. Phys. 51, 4150 (1980).

${ }^{15}$ D. Stievenard, X. Boddaert, J. C. Bourgoin, and H. J. von Bardeleben, Phys. Rev. B 41, 5271 (1990).

${ }^{16}$ D. Pons and J. Bourgoin, J. Phys. C 18, 3829 (1985).

${ }^{17}$ F. H. Eisen, K. Bachem, E. Klausman, K. Koehler, and R. Haddad, J. Appl. Phys. 72, 5593 (1992).

${ }^{18}$ J. C. Bourgoin, H. J. Von Bardeleben, and D. Stiévenard, J. Appl. Phys. 64, R65 (1988).

${ }^{19}$ M. Kaminska, M. Skowronski, and W. Kuszko, Phys. Rev. Lett. 55, 2204 (1985).

${ }^{20}$ H. H. Tan, J. S. Williams, and C. Jagadish, J. Appl. Phys. 78, 1481 (1995).

${ }^{21}$ G. M. Martin, P. Secordel, and C. Venger, J. Appl. Phys. 53, 8706 (1982).

${ }^{22}$ D. Stievenard, X. Boddaert, and J. C. Bourgoin, Phys. Rev. B 34, 4048 (1986)

${ }^{23}$ H. J. von Bardeleben, J. C. Bourgoin, and A. Miret, Phys. Rev. B 34, 1360 (1986). 